\title{
STRUTTURA DELLA CROSTA TERRESTRE IN CORRISPONDENZA DELL'ITALIA CENTRALE (*) (Gran Sasso)
}

\author{
D. Di Filippo - L. Marcelli
}

Richiami. - Il 5 settembre 1950, alle ore $04^{\mathrm{h}} 09^{\mathrm{m}}$ G.C.T. circa, ebbe luogo un terremoto nella zona del Gran Sasso d'Italia. Detto movimento tellurico, di notevole intensità, data la posizione dell'epicentro fu fatto oggetto di un particolare studio $\left({ }^{123}\right)$. Le coordinate epicentrali, il tempo origine $H$ e la profondità ipocentrale $h$ risultarono le seguenti :

$$
\begin{aligned}
\gamma^{\prime} & =42^{\circ} 30^{\prime}, 8 \pm 2^{\prime}, 0 \quad \mathrm{~N} \\
\lambda & =13^{\circ} 19^{\prime}, 6+5^{\prime}, 4 \quad \mathrm{E} \\
h & =5 \mathrm{~km} \\
H & =04^{\mathrm{h}} 08^{\mathrm{m}} 57^{\mathrm{s}}, 5 \pm 0^{\mathrm{s}}, 5
\end{aligned}
$$

In uno dei lavori accennati $\left({ }^{2}\right)$ abbiamo calcolato lo spessore dello strato del granito nell'Italia centrale valendoci dei risultati conseguiti per le velocità delle onde $P g$ e $P^{*}$. Applicando infatti la nota formula

$$
2 d=\frac{\frac{\Delta}{v_{\mathrm{P}_{\mathrm{g}}}}-\frac{\Delta}{v_{\mathrm{r}^{*}}}-\delta}{\sqrt{\left(\frac{1}{v_{\mathrm{P}_{\mathrm{g}}}}\right)^{2}-\left(\frac{1}{v_{\mathrm{p}^{*}}}\right)^{2}}}+h
$$

a sei stazioni cbe presentavano chiari esempi di onde $P^{*}$, prendendo $v_{\mathrm{P}_{\mathrm{w}}}=5,46 \mathrm{~km} / \mathrm{sec}, v_{\mathrm{P}^{*}}=6,38 \mathrm{~km} / \mathrm{sec}$; $h=5 \mathrm{~km}$ e per $\delta$ le differenze fra i tempi di registrazione delle $P g$ e delle $P^{*}$ in ogni stazione, abbiamo trovato che lo spessore medio $d$ dello strato del granito nell'Italia centrale è di $26 \mathrm{~km}$.

Spessore dello strato del granito con metodo grafico. - Abbiamo voluto calcolare lo spessore dello strato del granito anche sfruttando un metodo grafico in uso nella prospezione sismica del sottosuolo a

(*) Comunicazione presentata alla Riunione della Commissione Sismologica Europea dell'U.G.G.I, tenuta a Stoccarda nel settembre 1952. 
scopo minerario, e già adoperato con successo da Caloi (") per determinare lo strato del granito nel Cansiglio.

Questo metodo sfrutta i punti angolari nelle curve dei tempi, creati da brusche variazioni di velocità del raggio sismico, quando questo passa da uno strato ad un altro. Nel caso di una stratificazione piana a superfici parallele tra loro ed alla superficie esterna della terra (cosa che per piccole distanze può ritenersi vera in ơrni caso) le dromocrone ottenute registrando in un certo punto le onde provocate da uno scoppio artificiale, presenteranno un primo punto angolare in corrispondenza della brusca variazione di velocità determinata dall'arrivo in superficie delle onde che sono penetrate nel secondo strato (ovviamente nel caso di un terremoto questo avviene quan. do le $P g$ divengono $P^{*}$ penetrando nello strato del hasalto). A questo punto angolare corrisponde, sul terreno, il punto in cui arrivano contemporaneamente in superficie le onde longitudinali dirette $\left(P_{g}\right)$ e le onde longitudinali rifratte dalla superficie limitante inferiormente lo strato $\left(P^{*}\right)$. La profondità di quest'ultima superficie è data dalla profondita del punto in cui il raggio limite uscente dal centro di scoppio (cioè dall'ipocentro) interseca il luogo dei punti in cui i due sistemi di onde, diretto e rifratto, giungono contemporaneamente a distanze diverse dall'ipocentro, nell'interno dello strato. La costruzione di questo luogo geometrico si ottiene congiungendo i punti di intersezione di una schiera di rette (fronti delle onde rifratte) e del corrispondente fascio di cerchi (fronti delle onde dirette) concentrici nell'ipocentro.

Teoricamente si procede nel seguente modo (nel caso di un terremoto):

Si assuma l'epicentro come origine delle coordinate: l'asse $x$ si orienti dall'epicentro alla stazione di osservazione, e l'asse $\boldsymbol{z}$ dall'epicentro all'ipocentro. Il tempo origine sia quello del terremoto grià calcolato.

L'equazione dei fronti d'onda delle $P g$ (fascio di cerchi) sarà :

$$
x^{2}+(z-h)^{2}=v_{\mathrm{P}_{\mathrm{t}}} t^{2}
$$

( $h=$ profondità ipocentrale, $t=$ tempo di tragitto delle $P g, v_{\mathrm{lg}_{\mathrm{g}}}=$ velocità delle onde longitudinali dirette nel primo strato).

L'equazione dei fronti d'onda delle $P^{*}$ nell'interno dello strato delle $P g$ (schiera di rette) è:

$$
(z-d) \cos i-(x-\alpha) \sin i+v_{P_{i}}\left(t-t_{1}\right)=0
$$


( $d=$ spessore dello strato in esame, $i=$ angolo di incidenza del raggio limite, $a=$ ascissa del relativo punto d'incidenza sulla superficie di discontinuità, $t_{1}=$ tempo di tragitto dall'ipocentro al punto di incidenza). Eliminando $t$ tra le due equazioni si ottiene il luogo cercato: si ha:

$$
\begin{gathered}
(x \cos i+z \sin i)^{2}-x(2 d-h) \sin 2 i+2 z\left[(2 d-h) \cos ^{2} i-h\right] \\
-(2 d-h)^{2} \cos ^{2} i+h^{-}=0
\end{gathered}
$$

che è l'equazione di una parabola.

Per la costruzione grafica si procede nel modo seguente: le dromocrone delle $P g$ e delle $P^{*}$ (riferite al tempo origine del terremoto) si incontrano in un punto, cui corrisponde una certa distanza epicentrale (per noi $\Delta=162 \mathrm{~km}$ ). A questa distanza evidentemente le due onde giungono insieme e ciò avviene precisamente $29^{\mathrm{s}}, 8$ dopo il tempo origine del terremoto. La conoscenza delle velocità di propagazione delle $P g$ e delle $P^{*}$ ci consente la determinazione di $i$ :

$$
\operatorname{sen} i=\frac{v_{\mathrm{P}_{1}}}{v_{\mathrm{P}^{*}}}=5,46: 6.38 \quad i=\sim 58^{\circ} 5 \mathrm{I}^{\prime}
$$

Possiamo quindi tracciare (v. fig. 1) un conveniente numero di rette (fronti d'onda delle $P^{*}$ nell'interno del primo strato) inclinate dell'an-

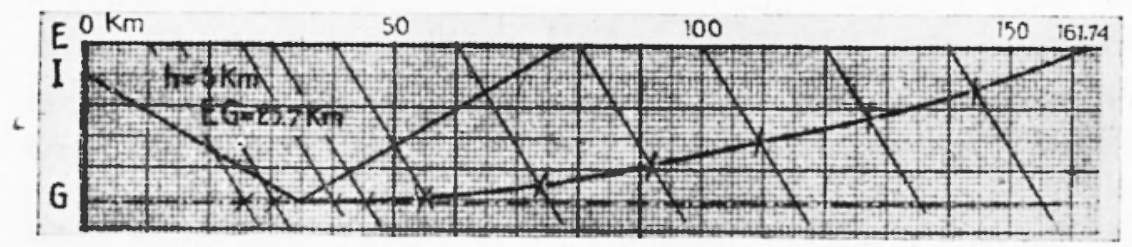

Fig. 1

golo $i$ rispetto alla superficie della terra. Intersecate queste rette con altrettanti cerchi (fronti d'onda delle $\mathrm{Pg}$ ) concentrici nell'ipocentro e raggi corrispondenti ai tempi relativi ad ogni singolo fronte d'onda, il luogo dei punti d'intersezione è la parabola cercata. Il punto in cui la parabola cosi costruita incontra il raggio limite uscente dall'ipocentro è un punto della superficie di separazione di due mezzi, cioc̀ rappresenta la profondità della prima superficie di discontinuitì. Dalla figura risulta evidente che l'intersezione avviene ad una profondità di $25,5 \mathrm{~km}$, in perfetto accordo con il precedente risultato. In- 
tendiamo nel presente lavoro calcolare la profondità della superficie di Mohorovicic, limitante inferiormente lo strato del basalto.

Calcolo dello spessore della crosta terrestre in corrispondenza del Gran Sasso d'Italia.

a) Tentativo con il metodo del raggio d'inversione. - Un primo tentativo per trovare questo spessore, lo abliamo fatto adottando il metodo segnalato dal giapponese Minakami $\left({ }^{4}\right)$.

Si parte dall'ipotesi che il fuoco del sisma si trovi nella crosta terrestre: si suppone inoltre che la distribuzione degli impulsi iniziali

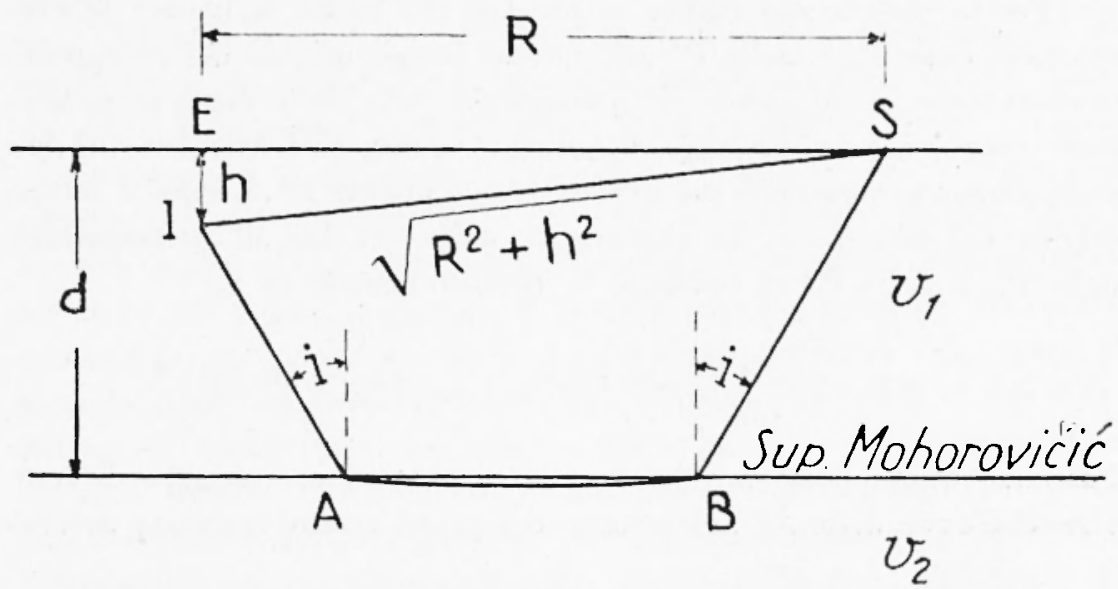

Fig. 2

del terremoto nelle varie stazioni sia tale da poter individuare con sufficiente precisione il raggio $R$ del cerchio d'inversione del segno dei movimenti iniziali (v. fig. 2).

Sia $h$ la profondità ipocentrale, $d$ lo spessore della crosta terrestre, $i$ l'angolo d'incidenza dell'onda sismica sulla superficie di discontinuità supposta parallela alla superficie esterna ritenuta piana: se $v_{1}$ e $v_{y}$ sono le velocità di propagazione delle onde longitudinali nel primo e nel secondo strato della crosta terrestre, con delle considerazioni ahluastanza semplici di eguaglianze di tempi di tragitto, si ha cle:

$$
\frac{\sqrt{k^{2}+h^{2}}}{v_{1}}=\frac{2 d-h}{v_{1} \cos i}+\frac{1}{v_{2}}-\{R-(2 d--h) \operatorname{tg} i\}
$$

Nel nostro caso il raggio $R$ d'inversione è ben individuato. Difatti nelle stazioni intorno all'epicentro il movimento iniziale si è presentato dovunque come una compressione fino a Padova $(\Delta=341 \mathrm{~km}$ ) 
menıre a Trieste $(\Delta=349 \mathrm{~km})$ e nelle stazioni più distanti è comparso come dilatazione $\left({ }^{1}\right)$. Senza timore di commettere un grosso errore, possiamo prendere $R=34.5 \mathrm{~km}$. Occorre però osservare che il caso contemplato da Minakami si riferisce ad un solo strato. Sappiamo invece che prima di giungere alla superficie di discontinuità di Mohorovicic le onde longitudinali percorrono lo strato del granito prima e quello del basalto poi, con velocità diverse: i valori da noi trovati sono precisamente: $v_{\mathrm{P}_{\mathrm{g}}}=5,46 \mathrm{~km} / \mathrm{sec}, v_{\mathrm{P}^{*}}=6,38 \mathrm{~km} / \mathrm{sec} \mathrm{e}$ infine $v_{\mathrm{Pn}_{\mathbf{n}}}=8,19 \mathrm{~km} / \mathrm{sec}$.

Abbiamo ritenuto lecito, seppure non rigorosamente esatto, mediare la velocità delle $P g$ e delle $P^{*}$ onde applicare la formula di Minakami ponendo

$$
v_{1}=5,92 \mathrm{~km} / \mathrm{sec} ; v_{2}=v_{\mathrm{P}_{11}=: 8,19 \mathrm{~km} / \mathrm{sec} .}
$$

Allora, lo spessore della crosta terrestre calcolato applicando la [1] risulta essere

$$
d \sim 70 \mathrm{~km} \text {. }
$$

Il valore eccessivo trovato non ci consente di accettare senz'altro il risultato conseguito: tuttavia può nascere il dubbio che lo spessore in esame possa in realtà essere più elevato di quanto non si pensi.

b) Metodo analitico per il calcolo degli strati. - Lo spessore dello strato del granito e di quello del basalto si può calcolare sfruttando la conoscenza delle dromocrone delle onde $P_{x_{1}}$ e $P x_{9}$. Per la teoria, abbastanza semplice del resto, rimandiamo al già citato lavoro di Caloi ( ${ }^{5}$. Ci limitiamo a riportare qui le formule di cui ci siamo serviti: se con $d_{1}$ indichiamo lo spessore dello strato del granito, con d. quello del basalto, con $t_{\mathrm{P}_{\mathrm{x}}} t_{\mathrm{P}_{\mathrm{x}}} t_{\mathrm{P}_{\mathrm{n}}}$, i tempi di arrivo delle $P x_{1}$, $P_{x_{2}}, P n$ nelle varie stazioni, con $v_{P_{y}}, \quad v_{S_{g}}, \quad v_{\mathrm{P}^{*},}, v_{P_{n}}, v_{\mathbf{S}^{*}}$. le velocità dei vari tipi d'onde sismiche, valgono le seguenti relazioni:

$$
\begin{gathered}
d_{1}=h+\frac{t_{\mathrm{Px}_{1}}-t_{\mathrm{Pa}}}{\sqrt{\left(\frac{1}{v_{\mathrm{S}_{\mathrm{g}}}}\right)^{2}-\left(\frac{1}{v_{\mathrm{P}_{\mathrm{n}}}}\right)^{2}-\sqrt{\left(\frac{1}{v_{\mathrm{P}_{\mathrm{g}}}}\right)^{2}-\left(\frac{1}{v_{\mathrm{P}_{\mathrm{n}}}}\right)^{2}}}} \\
d_{\mathrm{z}}=\frac{t_{\mathrm{Px}_{\mathrm{x}}}-t_{\mathrm{P}_{\mathrm{x}} 1}}{\sqrt{\left(\frac{1}{v_{\mathrm{S}^{*}}}\right)^{2}-\left(\frac{1}{v_{\mathrm{P}_{\mathrm{n}}}}\right)^{2}}-\sqrt{\left(\frac{1}{v_{\mathrm{P}^{*}}}\right)^{2}-\left(\frac{1}{v_{\mathrm{p}_{\mathrm{S}}}}\right)^{2}}}
\end{gathered}
$$

Prese le seguenti otto stazioni: Taranto, Cluur, Zürich, Wienn, Neuchâtel, Bàsel, Stuttgart, Jena, abliamo calcolato le differenze dei tempi di arrivo delle varie onde e abbiamo ottenuto $i$ seguenti valori 
medi : $t_{\mathrm{P}_{\mathbf{x}_{1}}}-t_{\mathrm{Pn}_{\mathrm{n}}}=3^{\mathrm{s}}, 4, t_{\mathrm{P}_{\mathbf{x}}}-t_{\mathrm{P}_{\mathrm{x}_{1}}}=5^{\mathrm{s}}, 25$. Sostituendo poi alle varie velocità $i$ valori da noi trovati nel precedente lavoro $\left({ }^{3}\right)$

$$
\begin{gathered}
v_{\mathrm{P}_{\mathrm{g}}}=5,46 \mathrm{~km} / \mathrm{sec} . v_{\mathrm{S}_{\mathrm{g}}}=3,01 \mathrm{~km} / \mathrm{sec} . v_{\mathrm{P}_{\mathrm{n}}}=8,19 \mathrm{~km} / \mathrm{sec} . \\
v_{\mathrm{P}^{*}}=6,38 \mathrm{~km} / \mathrm{sec} . \quad v_{\mathrm{S}^{*}}=3,63 \mathrm{~km} / \mathrm{sec} .
\end{gathered}
$$

abbiamo ottenuti $\mathrm{i}$ seguenti risultati:

$$
\begin{aligned}
& d_{1}=24,71 \mathrm{~km} \\
& d_{2}=36,02 \mathrm{~km}
\end{aligned}
$$

Il primo valore conseguito è in ottimo accordo con quelli trovati precedentemente con altri metodi, interessando altre onde; sicché possiamo ormai affermare con buona attendibilità cbe lo spessore dello strato del granito nell'Italia centrale è

$$
d_{\text {granito }} \sim 25 \mathrm{~km}
$$

Il valore $d$. dello strato del basalto a tutta prima ci è sembrato un po' troppo elevato sebbene già il tentativo con il raggio d'inversione ( $\$$ a) facesse intravedere tale risultato. Tuttavia abbiamo voluto cercare ulteriore conferma.

c) Conferma dei risultati conseguiti, mediante la dromocrona delle RMP. - Ci siamo valsi allo scopo, della dromocrona delle $R i P g$, da noi calcolata nel precedente lavoro $\left({ }^{3}\right)$. L'equazione di detta dromocrona, riferita al tempo origrine del terremoto risultò la sequente:

$$
t^{\prime \prime}=(0,03054 \pm 0,00073) \Delta^{2}+(425,8981+61,1066)
$$

All'epicentro (per $\Delta=0$ ) la curva taglia l'asse dei tempi nel punto (trascurando gli errori)

$$
t_{\mathrm{F}}=\sqrt{425,8981}=20^{\mathrm{s}, 6}
$$

E ben noto che si indicano con $R i P_{g}$ quelle onde longitudinali che si riflettono una volta sulla superficie di Mohorovicic e che noi chiameremo $R M P$. Calcoliamo dunque il tempo impiegato dalle onde longitudinali per tornare in superficie dopo essere partite dall'ipocentro $I$, situato ad una profondità $h=5 \mathrm{~km}$.

Ammettendo per buoni i valori degli spessori trovati nel paragrafo precedente si ha cbe le onde in questione viaggiano complessivamente per $(20+25) \mathrm{km}$ nello strato del granito con la velocità delle $\mathrm{Pg}\left(v_{\mathrm{P}}=5,46 \mathrm{~km} / \mathrm{sec}\right.$. $)$ e per $(36+36) \mathrm{km}$ nello strato del basalto con la velocità delle $P^{*}\left(v_{\mathrm{P}^{*}}=6,38 \mathrm{~km} / \mathrm{sec}\right.$. $)$. 
Se ora calcoliamo il tempo di tragitto delle $R M P$ si ha:

$$
t_{\mathrm{RMP}}=13^{\mathrm{s}}, 53
$$

Questo valore, confrontato con il $20^{5}, 6$ dato dalla dromocrona, offre una sorprendente conferma delle ipotesi assunte.

Discussione dei risultati. - Esaminando i risultati ottenuti nei precedenti paragrafi e tenendo conto dei vari metodi usati, ciascuno dei quali si serve di elementi diversi e indipendenti l'uno dall'altro, siamo portati ad accettare ormai per huoni $i$ valori trovati e ad asse-

Tabella 1

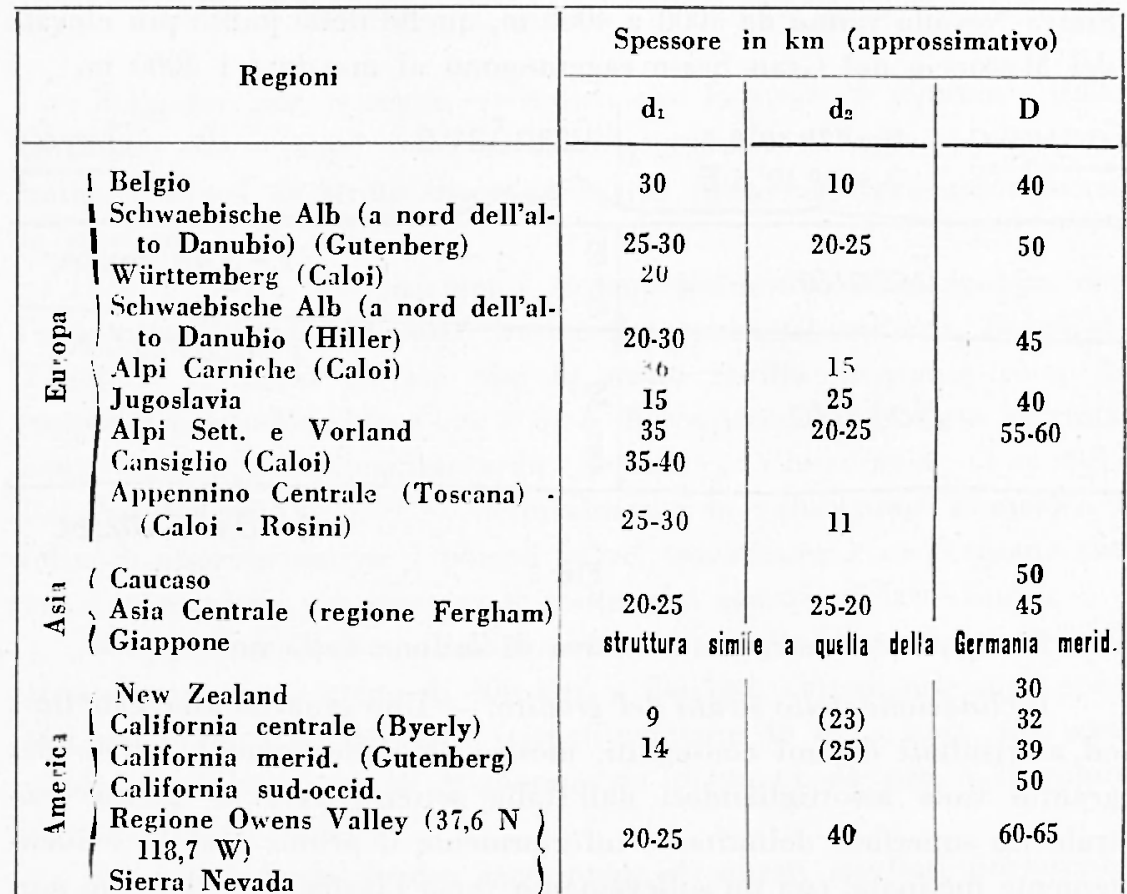

gnare alla crosta terrestre sotto il Gran Sasso, uno spessore complessivo di circa $60 \mathrm{~km}$. Questo valore, del resto, non è esagerato se lo si considera nel quadro generale degli spessori calcolati da altri autori per altre regioni.

La Tabella I riassume questi elementi $\left(^{6}\right)$ : (essendo $d_{1}=$ spessore dello strato del granito; $d_{2}=$ spessore dello strato del basalto; $D=$ profondità della superficie di Mohorovicic, ossia spessore totale della crosta terrestre).

Dalla tabella risulta chiaro come i massimi valori trovati per 
lo spessore della crosta terrestre siano in corrispondenza delle zone alpine in Europa e della Sierra Nevada in California, ma mentre sotto

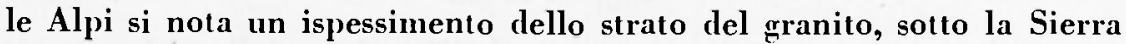
Nevada sembra che l'aumento complessivo dello spessore sia dovuto più sensibilmente ad un ispessimento dello strato del basalto.

Nel caso della zona appenninica del Gran Sasso, sembra quasi che ci si debha trovare in una situazione intermedia, giacché come si è visto

$$
d_{1} \sim 25 \mathrm{~km} \quad d_{: .} \sim 36 \mathrm{~km} \quad D \sim 60.61 \mathrm{~km}
$$

Notiamo incidentalmente che mentre le altitudini medie della Sierra Nevada vanno da 3000 a $4000 \mathrm{~m}$, quelle delle punte più elevate del Massiccio del Gran Sasso raggiungono al massimo i $3000 \mathrm{~m}$.

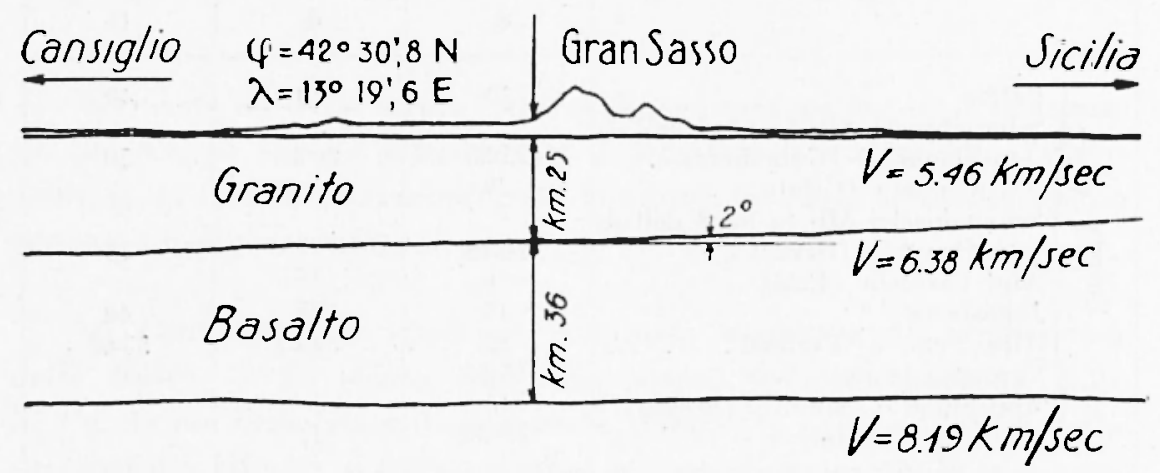

Fig. 3

La figura 3 mostra un tentativo di sezione della nostra zona.

Inclinazione dello strato del granito. - Uno sguardo alla Tabella I ed ai risultati da noi conseguiti, mostra evidente come lo strato del granito vada assottigliandosi dall'Italia settentrionale a quella centrale: la superficie delimitante inferiormente il primo strato è evidentemente inclinata, con un sollevamento verso l'Italia centrale e ciò non costituisce che una ulteriore conferma a quanto già da altri autori era stato detto in proposito $\left({ }^{7}\right)$.

Abliamo tentato di valutare l'ordine di grandezza dell'angolo di inclinazione, ed all'uopo ci siamo valsi del metodo che Caloi espone nel lavoro già citato $\left({ }^{7}\right)$. Da considerazioni ablhastanza semplici si perviene alle seguenti due formule che legano tra loro gli elementi di due qualsiansi stazioni sismiche allineate con l'epicentro:

$$
\sin (i \pm \lambda)=v_{1} \frac{t_{2}-t_{1}}{\Delta_{2}-\Delta_{1}}
$$


I simboli hanno $i$ seguenti significati:

$v_{1}=$ velocità delle onde longitudinali dirette: $v_{\mathrm{P}_{\mathrm{g}}}=5,46 \mathrm{~km} / \mathrm{sec}$. $t_{1}, t_{2}=$ tempi d'arrivo delle onde $P^{*}$ nelle due stazioni prescelte $\Delta_{1}, \Delta_{2}=$ distanze epicentrali delle due stazioni (in $\mathrm{km}$ )

$i \quad=$ angolo d'incidenza della $P_{g}$ sulla superficie in questione:

$$
\sin i=\frac{v_{i_{b}}}{v_{\mathrm{P}^{*}}} \quad i=58^{\circ} 52^{\prime} 40^{\prime \prime}
$$

$\lambda \quad=$ inclinazione della superficie di discontinuità rispetto alla superficie terrestre.

Nella formula il segno - indica che lo strato è montante dallo epicentro alle stazioni (cioè va assottigliandosi), il segno + viceversa indica trattarsi di strato discendente (lo strato va ispessendosi verso le stazioni).

Scelti alcuni accoppiamenti in approssimativo allineamento con l'epicentro (Taranto-Messina, Basilea-Stoccarda, Chur-Pavia, BelgradoTrieste...) abbiamo trovato che lo strato risulta montante verso la coppia Taranto-Messina $\left(\lambda \sim 2^{\circ}, 3\right)$ e discendente verso tutte le rimanenti coppie: Basilea-Stoccarda $\left(\lambda \sim 5^{\circ}, 9\right)$, Chur-Pavia $\left(\lambda \curvearrowright 4^{\circ}, 1\right)$, Belgrado-Trieste $\left(\lambda \curvearrowright 2^{\circ}, 5\right)$. Naturalmente la valutazione numerica è soltanto approssimativa. I piccoli valori trovati per $/$ confermano tuttavia in maniera più rigorosa le ipotetiche asserzioni fatte finora.

Se poi consideriamo l'allineamento Cansiglio-Gran Sasso ile due zone distano poco meno di $400 \mathrm{~km}$ ) e fissiamo l'attenzione sugli spessori del granito ivi calcolati (rispettivamente 40 e $25 \mathrm{~km}$ ), una semplicissima valutazione di trigonometria piana ci dà per $\lambda$ il valore approssimativo di $2^{\circ}, 2$.

La concordanza invero eccezionale di questi risultati porterebbe inoltre a desumere che scendendo ulteriormente verso l'Italia meridionale, ad un certo punto il granito dovrebbe addirittura scomparire (a circa $1000 \mathrm{~km}$ dal Consiglio in allineamento meridiano). È naturalmente azzardato affermare senz'altro una cosa simile: sta di fatto però, che a detta di alcuni geologi, nell'isola di Ustica (a nord della Sicilia, circa $850 \mathrm{~km}$ a sud del Cansiglio) in alcuni punti affiora il basalto.

Roma - Istituto Nazionale di Geofisica - Agosto 1952. 


\section{RIASSUNTO}

Sulla base dei risultati conseguiti nello studio del terremoto avvenuto il 5 settembre 1950 con epicentro nella zona montuosa del Gran Sasso d'Italia, sono stati effettuati vari calcoli per tentare di individuare lo spessore dello strato del granito e la profondità della superficie di Mohorovicic. I risultati cui si è giunti sono oltremodo interessanti: vari metodi applicati (metodo del raggio del cerchio di inversione degli impulsi iniziali, metodo grafico delle superfici d'onda, delle $\mathrm{Pg}_{\mathrm{g}} \mathrm{e} \mathrm{P}^{*}$, metodo analitico con le dromocrone delle $\mathbf{P x}_{1}$ e $\mathbf{P x}_{\mathbf{x}_{2}}$, conferma con la dromocrona della RMP), hanno condotto, con sorprendente uniformità, ad assegnare allo strato del granito per l'Italia centrale uno spessore di $25 \mathrm{~km}$, ed a quello del basalto uno spessore di $36 \mathrm{~km}$, sicché la superficie di Mohorovicic si troverebbe ad una profondità di $\sim 60 \mathrm{~km}$, paragonabile alle profondità trovate nelle zone alpine (Carnia meridionale, Vorland...) e nella Sierra Nevada.

E stato inoltre accertato che la superficie alla base dello strato del granito risulta inclinata di un piccolo angolo (compreso tra $2^{\circ}$ e $5^{\circ}$ ), il che determina un assottigliamento del suddetto strato verso l'Italia meridionale.

\section{SUMMARY}

On the basis of the results obtained in the study of the earthquake which occured September 5, 1950 with epicenter in the mountainous zone of Gran Sasso, Italy, various calculations have been made to try to determine the thickness of the layer of granite and the depth of the surface of Mohorovicic. The results thus obtained are interesting for other reasons. The various methods applied (method of the radius of curvature of inversion of the initial impulses, graphical method on the wave surfaces $\mathrm{Pg}_{\mathrm{g}}$ and $\mathrm{P}^{*}$, analytic method with the travel times of $\mathrm{Px}_{1}$ and $\mathrm{Px}:$, checkd with the travel time of $\mathrm{RMP}$, have lead, with surprising uniformity to a value of $25 \mathrm{~km}$ for the thickness of the layer of granite for Central Italy, and for the basalt layer a thickness of $36 \mathrm{~km}$, so that the surface of Mohorovicic is at a depth of about $60 \mathrm{~km}$, comparable to that found in the Alpine zone (Central Carnia, Vorland...) and in the Sierra Nevada.

It has been further found that the surface at the botton of the layer of granite is inclined at a smallangle (betueen $2^{\circ}$ and $5^{\circ}$ ), 
and this produces a diminution of the above-mentioned layer toward central Italy.

\section{BIBLIOGRAFIA}

(1) D. Di Filippo, L. Marcelli: Uno studio del terremoto del Gran Sasso d'Italia del 5 settembre 1950. “Annali di Geofisica " IV, n. 2 (1951).

(-) D. Di Filippo, L. Marcelli: Tempi di tragitto delle onde $P^{*}$ e spessore dello strato del granito nell Italia Centrale. “Annali di Geofisica » IV, n. 4 (1951).

(3) D. Di Frlippo, L. Marcelli: Dromocrone per terremoti vicini e velocità delle onde nellitalia Centrale. "Annali di Geofisica 》 V, n. 2 (1952).

(4) Minakami T.: Distribution des moviments initiaux d'un seisme dont le foyer se trouve, dans la couche superficielle, et determination de l'épaisseur de rette couche. "Bulletin Earthq. Research Institut», Tokio Imperial University, vol. XIII, 1935.

(5) P. Calor: Ricerche su terremoti ad origini vicine. Scosse del Cansiglio dell'ottobre 1936. "Ricerca Scientifica », vol. II n. 7-8.

(6) P. Calor: Sulla velocità di propagazione delle onde $p^{*}$ e sullo spessore dello strato del granito nell'Europa centrale. "La Ricerca Scientifica ", anno XI, n. 11 (novembre 1940).

(7) B. GutenberG: Seismological evidence for roots of mountains. "Bull. Geolog. Soc. of America. Vol. 54, aprile 1943. 